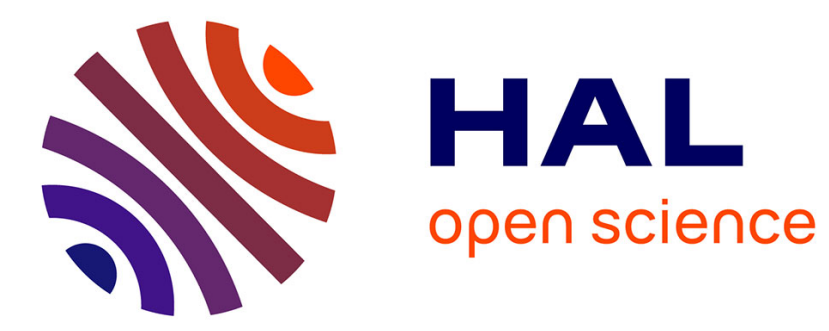

\title{
A dynamic regularized gradient model of the subgrid-scale scalar flux for large eddy simulations
}

Guillaume Balarac, Julien Le Sommer, Xavier Meunier, Antoine Vollant

\section{To cite this version:}

Guillaume Balarac, Julien Le Sommer, Xavier Meunier, Antoine Vollant. A dynamic regularized gradient model of the subgrid-scale scalar flux for large eddy simulations. Physics of Fluids, 2013, pp.075107. 10.1063/1.4813812 . hal-00835655

\section{HAL Id: hal-00835655 https://hal.science/hal-00835655}

Submitted on 19 Jun 2013

HAL is a multi-disciplinary open access archive for the deposit and dissemination of scientific research documents, whether they are published or not. The documents may come from teaching and research institutions in France or abroad, or from public or private research centers.
L'archive ouverte pluridisciplinaire HAL, est destinée au dépôt et à la diffusion de documents scientifiques de niveau recherche, publiés ou non, émanant des établissements d'enseignement et de recherche français ou étrangers, des laboratoires publics ou privés. 


\title{
A dynamic regularized gradient model of the subgrid-scale scalar flux for large eddy simulations.
}

\author{
G. Balarac ${ }^{1}$, J. Le Sommer ${ }^{1}$, X. Meunier ${ }^{1}$ and A. Vollant ${ }^{1}$ \\ ${ }^{1}$ Grenoble-INP / CNRS / UJF-Grenoble 1, LEGI UMR 5519, Grenoble, F-38041, France
}

(Dated: April 26, 2013)

\begin{abstract}
Accurate predictions of scalar fields advected by a turbulent flow is needed for various industrial and geophysical applications. In the framework of large-eddy simulation (LES), a subgrid-scale (SGS) model for the subgrid-scale scalar flux has to be used. The gradient model, which is derived from a Taylor series expansions of the filtering operation is a well-known approach to model SGS scalar fluxes. This model is known to lead to high correlation level with the SGS scalar flux. However, this type of model can not be used in practical LES because it does not lead to enough global scalar variance transfer from the large to the small scales. In this work, a regularization of the gradient model is proposed based on a physical interpretation of this model. The impact of the resolved velocity field on the resolved scalar gradient is decomposed into compressional, stretching and rotational effects. It is shown that rotational effect is not associated with transfers of variance across scales. Conversely, the compressional effect is shown to lead to forward transfer, whereas the stretching effect leads to back-scatter of scalar variance. The proposed regularization is to neglect the stretching effect in the model formulation. The accuracy of this regularized gradient model is tested in comparison with direct numerical simulations (DNS) and compared with other classic SGS models. The accuracy of the regularized gradient model is evaluated in term of structural and functional performances, i.e. the model ability to locally approximate the SGS unknown term and to reproduce its global effect on tracer variance, respectively. It is found that the regularized gradient model associated with a dynamic procedure exhibits good performances in comparison with the standard dynamic eddy diffusivity model and the standard gradient model. In particular, the dynamic regularized gradient model provides a better prediction of scalar variance transfers than the standard gradient model. The dynamic regularized gradient model is then evaluated in a series of large-eddy simulations. This shows a substantial improvement for various scalar statistics predictions.
\end{abstract}




\section{INTRODUCTION}

Various applications need to solve a scalar equation simultaneously with the governing flow equations. In these applications, the scalar can represent the concentration of chemical species or the temperature field in mixing or heat transfer studies. Due to the large range of motion scales in turbulent flows, the direct numerical simulation of realistic applications is not yet feasible because of the high computational cost. To overcome this limitation, the large-eddy simulation technique proposes to explicitly solve only the large scales of the flow and to model the impact of the smallest scales on the large scales. Large eddy simulation methods encompass a broad range of applications, including turbulent combustion ${ }^{1}$ or geophysical fluids and in particular the simulation of ocean mesoscale flows ${ }^{2}$. In LES methods, a separation between resolved large scales and modeled small scales is performed by a filtering operation

$$
\bar{f}(\vec{x}, t)=\int f(\vec{y}, t) G(\vec{x}-\vec{y}) d \vec{y}
$$

to obtain the large-scale resolved field, $\bar{f}$, from the turbulent field, $f$, with $G$ the filter kernel. This filtering operation applied to the flow equations leads to subgrid-scale (SGS) terms which have to be modeled. While many SGS models have been designed to close the filtered Navier-Stokes equations for incompressible flows ${ }^{3-5}$, the corresponding problem for the scalar equation has not yet been fully addressed. The filtered transport equation for a passive scalar, $Z$, in incompressible flow is given by

$$
\frac{\partial \bar{Z}}{\partial t}+\bar{u}_{i} \frac{\partial \bar{Z}}{\partial x_{i}}=D \frac{\partial^{2} \bar{Z}}{\partial x_{i}^{2}}-\frac{\partial T_{i}}{\partial x_{i}}
$$

where $T_{i}=\overline{u_{i} Z}-\bar{u}_{i} \bar{Z}$ is the SGS scalar flux, which has to be modeled in the context of LES simulations. Two major strategies exist for developing SGS models ${ }^{5}$ : functional and structural strategies. The functional modeling strategy considers the action of the subgrid terms on the transported quantity and not the unknown term itself. It can introduce a dissipative term, for example, that has a similar effect but not necessarily the same spatial structure. Conversely, the structural modeling strategy consists of using the best local approximation of the unknown SGS term by constructing it from the known structure of small-scales. In the LES of passive scalar context, a typical functional model is to introduce an eddy diffusivity, $D_{T}$, to model the SGS scalar flux as $T_{i}=D_{T} \partial \bar{Z} / \partial x_{i}$. In the simplest models, the eddy diffusivity is defined from the eddy viscosity, $\nu_{T}$, through a constant eddy 
Schmidt number ${ }^{6}, S c_{T}=\nu_{T} / D_{T} \approx 0.6$. An important limitation of such a model if that $S c_{T}$ is set to a constant while it should be strongly affected by the slope of the kinetic energy spectrum $^{7}$. Models have been proposed to take into account the influence of the molecular Schmidt number and of the local turbulence level of the flow. For example, corrections have been proposed based on the eddy-damped quasi normal Markovian (EDQNM) theory ${ }^{8}$ or by using a dynamical procedure ${ }^{9}$. Thus, a dynamic model for $D_{T}$ is now often used, similarly to the dynamic Smagorinsky model of the eddy viscosity ${ }^{10}$. This dynamic eddy diffusivity model (noted DEDM) is defined as ${ }^{9}$

$$
T_{i}^{\mathrm{DEDM}}=D_{T} \frac{\partial \bar{Z}}{\partial x_{i}}=C \bar{\Delta}^{2}|\bar{S}| \frac{\partial \bar{Z}}{\partial x_{i}},
$$

where $\bar{\Delta}$ is the filter width and $|\bar{S}|=\left(2 \bar{S}_{i j} \bar{S}_{i j}\right)^{1 / 2}$, with $\bar{S}_{i j}=1 / 2\left(\partial \bar{u}_{i} / \partial x_{j}+\partial \bar{u}_{j} \partial x_{i}\right)$, the filtered strain rate tensor. The model coefficient $C$ is determined dynamically using the Germano identity ${ }^{10,11}$. Moin et al. ${ }^{9}$ show that the dynamic procedure greatly improves the results of the simulations and leads to a non constant value of $S c_{T}$. However, even if a correct dissipation level is modeled, DEDM is generally known to exhibit weak local correlations between the model and the SGS terms ${ }^{12}$. This behavior is typical of models derived with the functional strategy based on an eddy diffusivity model. Indeed, these models assume an alignment between the resolved scalar gradient and the SGS scalar flux, which is not observed ${ }^{13}$. To correct this behavior, various approach can be proposed based on the definition of a tensor-eddy diffusivity from the SGS stress tensor ${ }^{14}$, a scale-similarity assumption $^{15,16}$ or a Taylor series expansion of the filtering operation ${ }^{17}$.

Models defined from a Taylor series expansion of the filtering operation are typical structural models, often called gradient models ${ }^{12}$. In the case of the SGS scalar flux, this model writes as,

$$
T_{i}^{\mathrm{GM}}=\frac{\bar{\Delta}^{2}}{12} \frac{\partial \bar{u}_{i}}{\partial x_{j}} \frac{\partial \bar{Z}}{\partial x_{j}} .
$$

A similar model has also been introduced in the context of two dimensional geophysical fluid dynamics ${ }^{18}$. This type of model is known to provide a good local approximation of the unknown term with a high correlation between the unknown term and the model in a priori tests. However, this type of model is known to be unstable due to an incorrect prediction of the dissipation ${ }^{19}$. Thus, in a recent work, Lu and Porté-Agel ${ }^{20}$ propose a new SGS scalar flux model based on the gradient model, with a control of the magnitude of the SGS scalar flux. However, a clipping procedure is still needed to ensures a non-negative 
dissipation. Another popular solution to overcome the limitations of both functional and structural models is to use a mixed model. Thus, the concept of the Clark model consists in adding a gradient model to an eddy diffusivity model to obtain both the relatively accurate representation of the SGS term by the gradient model, and a proper dissipation provided by the eddy diffusivity mode ${ }^{12}$. A dynamic procedure can then be applied to define a dynamic Clark model ${ }^{21,22}$.

In this paper, we propose to explore another way to stabilize the gradient model. Thus, we develop a model combining the advantage of both approaches: structural and functional models. The approach is based on a physical interpretation of the terms involved in the gradient model. It is shown that only the stretching effects of the resolved velocity field on the resolved scalar gradient lead to scalar variance transfer from small to the large scales (backscatter effect). By neglecting the stretching effects, a regularization of the gradient model is then proposed. The proposed regularization is evaluated with both a priori tests (i.e. in comparison with SGS terms evaluated from a DNS database) and a posteriori tests (i.e. with fully prognostic large eddy simulations). From a priori tests, the regularized gradient model associated with a dynamic procedure is shown to exhibit very good performances. This dynamic regularized gradient model is then tested in a posteriori (LES) tests. From the LES performed, it is shown that the new model substantially improves the prediction of various scalar statistics in comparison with a classic dynamic eddy diffusivity model.

\section{REGULARIZATION OF THE GRADIENT MODEL}

\section{A. Functional performance of the gradient model}

The gradient model (GM) is a classic structural-based model coming from a Taylor series expansion of the filtering operator ${ }^{17}$. This model is known to have a high structural performance as a high correlation level with the SGS unknown term, but a weak functional performance with an over-estimation of back-scatter effects, leading to unstable simulation ${ }^{19}$. The functional performance of a SGS scalar flux model is given by its capability to well reproduce the grid-scales/subgrid-scales (GS/SGS) transfer between the resolved scalar variance, $\bar{Z}^{2}$, and the SGS scalar variance, $\overline{Z^{2}}-\bar{Z}^{2}$. This transfer is controlled by the SGS scalar dissipation rate $^{23,24},-T_{i} \partial \bar{Z} / \partial x_{i}$. This term is usually positive on average, and local negative 
value characterizes an inverse transfer (back-scatter). For an accurate LES, a model must reproduce the average SGS scalar dissipation rates correctly ${ }^{25}$. Various modifications have therefore been proposed to stabilize the gradient model and improve its functional performance. These modifications consist to use a "clipping" 20,21 or to combine this model with an eddy diffusivity model as in the mixed model ${ }^{12}$. Here, an alternate approach is proposed on the basis of an analysis of the GS/SGS transfer predicted by the gradient model.

\section{B. SGS scalar dissipation predicted by the gradient model}

In this section, we show that the gradient of the resolved velocity field governs the GS/SGS transfer predicted by the gradient model. More precisely, we show that the the GS/SGS transfer predicted by the gradient model depend on the spatial orientation of the velocity gradients. The starting point is to decompose the velocity gradient as $\partial \bar{u}_{i} / \partial x_{j}=\bar{S}_{i j}+\bar{\Omega}_{i j}$, with $\bar{S}_{i j}=1 / 2\left(\partial \bar{u}_{i} / \partial x_{j}+\partial \bar{u}_{j} / \partial x_{i}\right)$ and $\bar{\Omega}_{i j}=1 / 2\left(\partial \bar{u}_{i} / \partial x_{j}-\partial \bar{u}_{j} / \partial x_{i}\right)$, the filtered strain rate tensor and the filtered rotation rate tensor, respectively. The gradient model, Eq. (4), can thus be rewritten as

$$
T_{i}^{\mathrm{GM}}=\frac{\bar{\Delta}^{2}}{12}\left(\bar{S}_{i j}+\bar{\Omega}_{i j}\right) \frac{\partial \bar{Z}}{\partial x_{j}}
$$

allowing a decomposition of the gradient model as the strain and the rotational effects of the filtered velocity fields on the filtered scalar gradient. The filtered strain rate tensor being symmetric, it can further be decomposed as

$$
\bar{S}_{i j}=\sum_{k=1}^{3} \lambda^{(k)} e_{i}^{(k)} e_{j}^{(k)}
$$

with $\lambda^{(k)}$ and $\vec{e}^{(k)}$ the (real) eigenvalues and the unitary eigenvectors of the filtered strain rate tensor, respectively. The gradient model can therefore be written as,

$$
T_{i}^{\mathrm{GM}}=\frac{\bar{\Delta}^{2}}{12}\left(\bar{S}_{i j}^{\oplus}+\bar{S}_{i j}^{\ominus}+\bar{\Omega}_{i j}\right) \frac{\partial \bar{Z}}{\partial x_{j}}
$$

with

$$
\bar{S}_{i j}^{\ominus}=\sum_{k=1}^{3} \min \left(0, \lambda^{(k)}\right) e_{i}^{(k)} e_{j}^{(k)}
$$

and

$$
\bar{S}_{i j}^{\oplus}=\sum_{k=1}^{3} \max \left(0, \lambda^{(k)}\right) e_{i}^{(k)} e_{j}^{(k)} .
$$


In order to better understand the GS/SGS transfer predicted by the gradient model, the SGS scalar dissipation can be rewritten from the above decomposition of the gradient model,

$$
\begin{aligned}
-T_{i}^{\mathrm{GM}} \frac{\partial \bar{Z}}{\partial x_{i}} & =-\frac{\bar{\Delta}^{2}}{12}\left(\bar{S}_{i j}+\bar{\Omega}_{i j}\right) \frac{\partial \bar{Z}}{\partial x_{j}} \frac{\partial \bar{Z}}{\partial x_{i}} \\
& =-\sum_{k=1}^{3} \lambda^{(k)} e_{i}^{(k)} e_{j}^{(k)} \frac{\partial \bar{Z}}{\partial x_{j}} \frac{\partial \bar{Z}}{\partial x_{i}}-\bar{\Omega}_{i j} \frac{\partial \bar{Z}}{\partial x_{j}} \frac{\partial \bar{Z}}{\partial x_{i}} \\
& =-\sum_{k=1}^{3} \lambda^{(k)}\left(\frac{\partial \bar{Z}}{\partial x_{i}} e_{i}^{(k)}\right)^{2} .
\end{aligned}
$$

Indeed, it can be shown that $\bar{\Omega}_{i j}\left(\partial \bar{Z} / \partial x_{j}\right)\left(\partial \bar{Z} / \partial x_{i}\right)=0$. Thus, the transfer given by the gradient model is only controlled by the sign of the eigenvalues of $\bar{S}_{i j}$ and the SGS dissipation predicted by the gradient model can be written as,

$$
-T_{i}^{\mathrm{GM}} \frac{\partial \bar{Z}}{\partial x_{i}}=\frac{\bar{\Delta}^{2}}{12}(-\underbrace{\bar{S}_{i j}^{\oplus} \frac{\partial \bar{Z}}{\partial x_{j}} \frac{\partial \bar{Z}}{\partial x_{i}}}_{<0} \underbrace{-\bar{S}_{i j}^{\ominus} \frac{\partial \bar{Z}}{\partial x_{j}} \frac{\partial \bar{Z}}{\partial x_{i}}}_{>0}) .
$$

Since, the negative eigenvalues correspond to the compressional effects and the positive ones correspond to stretching effects ${ }^{26}$, a physical interpretation of the gradient model can thus be proposed in terms of compressional, stretching and rotational effects of the resolved velocity field on the resolved scalar gradient. Note that the rotational effect does not lead to GS/SGS transfer. Conversely, the compressional effect leads to forward transfer (positive value of the SGS dissipation), whereas the stretching effect leads to back-scatter (negative value of the SGS dissipation).

\section{Physical illustration of the velocity gradient effect on the GS/SGS transfer}

A physical illustration can be provided to show the compressional and stretching effects on the GS/SGS transfer, if we consider a simple flow configuration. We consider thus a passive scalar $Z$ seeded as a gaussian patch of scalar centered at $(x, y)=(0,0)$, in a flow defined by a pure stretching along the $x$-axis and pure compression along the $y$-axis. The velocity field has the form $(u, v)=(\alpha x,-\alpha y)$ with $\alpha>0$. For this case, the evolution of the scalar distribution is obtained analytically with the method of characteristics. Obviously, the initial scalar patch will be elongated along the $x$-axis and compressed along the $y$-axis as time goes, Fig. 1 (a). This flow configuration allows us to decompose the velocity gradient 


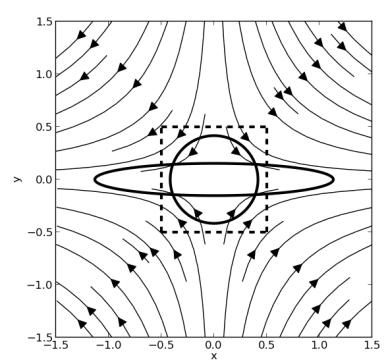

(a) stretching and compression

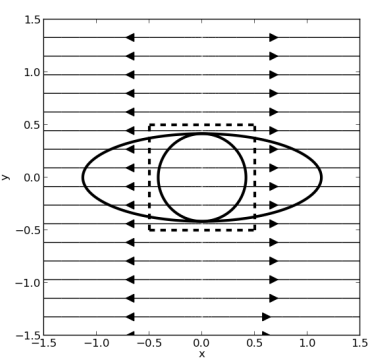

(b) pure stretching

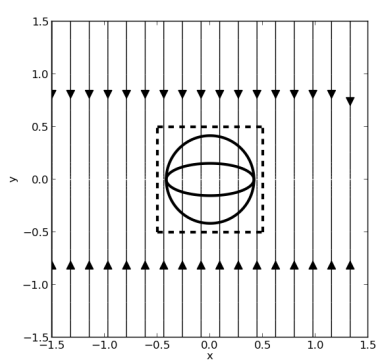

(c) pure compression

FIG. 1. Schematic of the three two-dimensional flow cases. The contour $Z(x, y, t)=\frac{1}{2} Z(0,0,0)$ is shown for $t=0$ (circle) and for $t=\alpha^{-1}$ for the stretching-compression case (a), the pure stretching case (b) and the pure compression case (c). The square box in dashed line is the center box where the SGS variance is computed as $\overline{Z^{2}}-\bar{Z}^{2}$, by using a box filter.

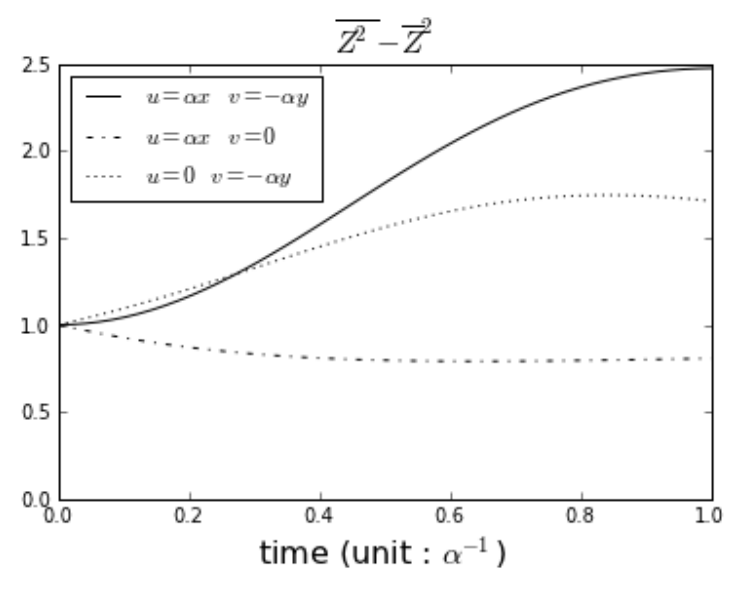

FIG. 2. Time series of SGS tracer variance in the center box for the three different flow cases: compression and stretching (plain line), stretching only (dash-dotted line) and compression only (dotted line). The initial condition is identical in all three cases. The time series are normalized by the initial value at $t=0$.

effect in taking into account only the stretching, $(u, v)=(\alpha x, 0)$, or only the compressional, $(u, v)=(0,-\alpha y)$, effects on the patch of scalar. If only the pure stretching part of the flow is retained to advect the scalar, the scalar patch scale increases with time, Fig. 1 (b). In contrast, if only the pure compressional part of the flow is retained,the scalar patch scale decrease with time, Fig. 1 (c). These scales generations will control the time evolution 
of the SGS scalar variance, $\overline{Z^{2}}-\bar{Z}^{2}$. In this case, the filtering operation consists in a box filter with $\bar{\Delta}=1$, as illustrated by the dashed line in Fig. 1. Figure 2 shows the time-evolution of this SGS variance computed in the center box. As expected, the SGS variance for $(u, v)=(\alpha x,-\alpha y)$ (plain line) increases with time characterizing a forward transfer from the resolved to the SGS scalar variance. Moreover, we can observed that pure compression, $(u, v)=(0,-\alpha y)$ (dotted-line), also leads to an increase in SGS variance, while pure elongation, $(u, v)=(\alpha x, 0)$ (dash-dotted line), leads to a moderate decrease of SGS variance in the center box (back-scatter). The evolution of SGS variance in this simple flow configuration is therefore consistent with the predictions of the gradient model. Indeed, the velocity gradients here control the GS/SGS transfer with compressional effects favoring direct transfers and stretching effects favoring indirect transfers (backscatter).

\section{Proposed regularizations}

As stated above, the gradient model is known to have a good correlation with the SGS scalar flux but this model lead to unstable simulation ${ }^{27}$. A regularization of this model that should allow keeping the high correlation level and would avoid the unstable behavior could lead to an accurate model for LES. The unstable behavior of this model is due to an overestimation of transfers from the subgrid scales to the grid scales by the model. To avoid this unstable behavior, our proposition is to neglect all these inverse transfers. From the previous section, this can be done by neglecting the stretching effects. At this stage, two models can be proposed. The first one is obtained by keeping only the term allowing the direct energy transfer. This leads to the first regularized gradient model (noted RGM1), which is written as

$$
T_{i}^{\mathrm{RGM} 1}=\frac{\bar{\Delta}^{2}}{12} \bar{S}_{i j}^{\ominus} \frac{\partial \bar{Z}}{\partial x_{j}} .
$$

Another proposition is only to neglect the term $\bar{S}_{i j}^{\oplus}$ in the gradient model decomposition, Eq. (6). The second regularized gradient model (noted RGM2) writes then

$$
T_{i}^{\mathrm{RGM} 2}=\frac{\bar{\Delta}^{2}}{12}\left(\bar{S}_{i j}^{\ominus}+\bar{\Omega}_{i j}\right) \frac{\partial \bar{Z}}{\partial x_{j}} .
$$

These two models differ only by keeping or not the rotational effect. As already seen, the rotational effect does not influence on the GS/SGS transfer instantaneously. These models will thus lead exactly to the same same GS/SGS transfer at a given time. Only the local 
approximation of the SGS scalar flux will be influenced by the rotational effect in the model formulation. This can be related to the bidimensional model proposed by Le Sommer at $a l .{ }^{28}$ for ocean circulation models. Our regularization approach is also close to the approach proposed by Cottet and Wray ${ }^{29}$ to develop an anisotropic model for the SGS shear stresses.

\section{MODELS PERFORMANCE MEASUREMENTS BASED ON A PRIORI TESTS}

\section{A. A priori evaluation of the regularized gradient models RGM1 and RGM2}

To better understand the advantage to use a regularized form for the gradient model, models performance is now evaluated from a priori tests. These tests are meant to measure the functional and structural performances of RGM1 and RGM2 and to compare these performances to classic models: the dynamic eddy diffusivity model (DEDM) and the gradient model (GM), given by Eq. (3) and Eq. (4), respectively. The a priori tests are based on data extracted from direct numerical simulation (DNS) of a forced homogeneous isotropic turbulence. The DNS database is generated from a standard pseudo-spectral code and the simulation domain is discretized using $512^{3}$ grid points on a domain of length $2 \pi$. A statistical steady flow is achieved by using a forcing term ${ }^{30}$ and the scalar field is initialized between 0 and 1 according to the procedure proposed by Eswaran and Pope ${ }^{31}$. The scalar is thus initialized as large spots with a size in order to the half of the computational box size. The Schmidt number is taken equal to 0.7 and the Reynolds number based on the Taylor microscale is around 180 at the stationary state. The code and the flow configuration are similar to previous works where the modeling of the SGS scalar flux ${ }^{22}$, the SGS scalar variance $^{32}$ and the SGS scalar dissipation rate ${ }^{33}$ were studied. The DNS data are filtered in space to emulate LES quantities by using a spectral cut-off filter to reproduce the behavior of the spectral method employed in this work. Several filter sizes have been used chosen as $2 \leq \bar{\Delta} / \Delta x \leq 16$, where $\bar{\Delta}$ is the filter width and $\Delta x$ is the DNS mesh size. Figure 3 shows the scalar variance spectrum with the location of the filters in the wavenumber space.

The functional performance is first studied. As defined by Sagaut ${ }^{5}$, the functional performance measures the ability of the model to reproduce the averaged effect of the SGS term on the transported quantity (here, the scalar field) and not the term itself. As already discussed, in the context of LES of a passive scalar, the functional performance is the ability 


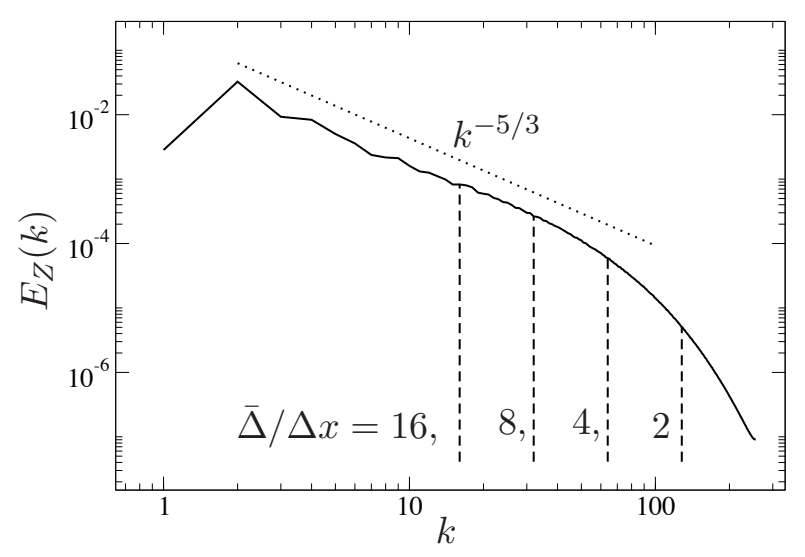

FIG. 3. Scalar variance spectrum, $E_{Z}(k)$, with the location of the filters used in the a priori tests.

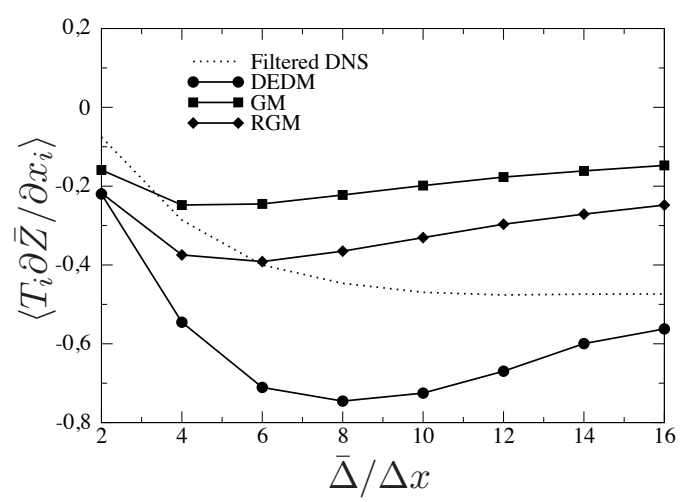

FIG. 4. Mean SGS dissipation as a function of the filter width for the regularization of the gradient model (RGM). The results for DEDM and GM are also shown. The dotted line show the SGS dissipation given by the filtered DNS data. Note that the SGS dissipation is the same for RGM1 and RGM2, Eq. (8) and (9), respectively.

to predict the GS/SGS transfer. This is controlled by the SGS scalar dissipation, $T_{i} \partial \bar{Z} / \partial x_{i}$. Figure 4 shows the mean SGS scalar dissipation, $\left\langle T_{i} \partial \bar{Z} / \partial x_{i}\right\rangle$, as a function of the filter width for various model. Note that RGM1 and RGM2 lead to the same SGS dissipation, noted RGM on the figure. The mean SGS scalar dissipation is negative showing that the transfers are from the large (resolved) scales to the small ones. First, as expected, the GM underpredicts the magnitude of $\left\langle T_{i} \partial \bar{Z} / \partial x_{i}\right\rangle$ in comparison with the DNS results. This shows that this model does not provide enough dissipation, leading to unstable simulations ${ }^{27}$. Con- 
versely, the DEDM models are too dissipative with an over-prediction of the magnitude of $\left\langle T_{i} \partial \bar{Z} / \partial x_{i}\right\rangle$. The regularization proposed allows to improve the prediction of the mean SGS scalar dissipation by neglecting the inverse transfer, but the magnitude of the SGS scalar dissipation is still under-predicted. In the following section, a dynamic procedure will be proposed to improve this prediction.

We now measure the structural performance of the regularized models in order to asses the performances of RGM1 and RGM2. From Sagaut ${ }^{5}$, the structural performance is defined as the model ability to describe locally the SGS unknown term appearing in the resolved equation. For the scalar, the SGS unknown term is the divergence of the SGS scalar flux, $\partial T_{i} / \partial x_{i}$, appearing in the scalar transport equation (2). In the framework of optimal estimation theory ${ }^{34}$, the models structural performance can be evaluated by using the notion of an optimal estimator recently introduced by Moreau et al. ${ }^{35}$ in the LES context. Considering $f$ as the SGS term to model and $g$ as a model of $f$ based on a given set of variables $\phi$, the quadratic error,

$$
\epsilon_{\mathrm{Q}}=\left\langle(f-g(\phi))^{2}\right\rangle
$$

is defined as the relevant error to consider in $\operatorname{LES}^{36}$. In this definition, the brackets indicate a statistical average over a suitable ensemble. The concept of optimal estimator forecasts that any model $g$ built on the set of variables $\phi$ will have a quadratic error higher than a minimal value, $\epsilon_{i r r}$. Moreover, this minimal value is defined by the optimal estimation theory as

$$
\epsilon_{i r r}=\left\langle(f-\langle f \mid \phi\rangle)^{2}\right\rangle \leq \epsilon_{\mathrm{Q}}
$$

where $\langle f \mid \phi\rangle$ is the expectation of the exact quantity $f$ conditioned with the set of variables $\phi$. The quantity $\langle f \mid \phi\rangle$ is thus called the optimal estimator of $f$ for the set of variable $\phi$, and the minimal error, $\epsilon_{i r r}$, is called the irreducible error since no model using only $\phi$ as set of variables can lead to a smaller error. The optimal estimator theory allows to provide various informations on the SGS models used in LES. First, we can evaluate the quadratic error of each model to see which one gives the best results in modelling the SGS unknown term. The most suitable set of variables to model the SGS term can also be determined by comparing the irreducible error of different models. The set of variables with the smallest irreducible error will be the best candidate to design a model. Finally, this theory allows to know to what extent a model based on a set of variable can be improved. 


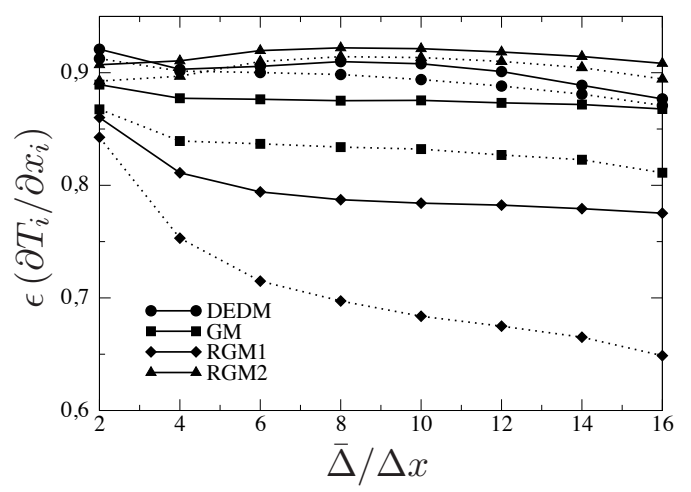

FIG. 5. Quadratic errors (QE) and irreducible errors (IE) as a function of the filter width for the regularization of the gradient model without (RGM1) and with (RGM2) the rotational term, as given by Eq. (8) and (9), respectively. The results for DEDM and GM are also shown. The dotted lines show the corresponding irreducible errors.

Indeed, if the quadratic error of a given model is much higher than its irreducible part, improvement can be expected (by modification of the coefficient computation, for example). This concept has already been used to improve the modeling of SGS quantities ${ }^{22,32,33}$. The optimal estimator tool is now used to measure the structural performance of the various models to model the divergence of the SGS scalar flux, $\partial T_{i} / \partial x_{i}$. Fig. 5 shows the evolution of the quadratic error, Eq. (10), and the irreducible error, Eq. (11), with the filter size. The errors are normalized by the statistical variance of the exact SGS term. For DEDM, the set of variables used to compute the irreducible error is $\left\{\frac{\partial}{\partial x_{i}}\left(|\bar{S}| \frac{\partial \bar{Z}}{\partial x_{i}}\right)\right\}$, whereas the GM set of variables is $\left\{\frac{\partial}{\partial x_{i}}\left(\frac{\partial \bar{u}_{i}}{\partial x_{j}} \frac{\partial \bar{Z}}{\partial x_{j}}\right)\right\}$. For the proposed regularized models, the set of variables used is $\left\{\frac{\partial}{\partial x_{i}}\left(\bar{S}_{i j}^{\ominus} \frac{\partial \bar{Z}}{\partial x_{j}}\right)\right\}$ for RGM1 and $\left\{\frac{\partial}{\partial x_{i}}\left(\left(\bar{S}_{i j}^{\ominus}+\bar{\Omega}_{i j}\right) \frac{\partial \bar{Z}}{\partial x_{j}}\right)\right\}$ for RGM2. Various conclusions can then be drawn. As expected the GM quadratic error is smaller than DEDM quadratic error. This is because GM is a structural based model, whereas DEDM is a functional based model. It is thus expected to have better structural performance. Moreover, the DEDM irreducible error is always higher than the GM quadratic error. This shows that the improvement of the structural performance of DEDM can not be expected without adding new quantities in its set of variables. For both proposed regularized gradient model (RGM1 and RGM2), the performances are very different. RGM1 has good structural performance conversely to 
RGM2. Indeed, the RGM2 irreducible error is even higher than DEDM quadratic error, showing that this model proposition does not allow an improvement in comparison with a classic eddy diffusivity model. Conversely, RGM1 performs even better than GM, with the RGM1 quadratic error smaller than the GM irreducible error, showing that we can not expect the same performance with the classic gradient model. However, an improvement can be expected for this model because the gap between the RGM1 quadratic error and its associated irreducible error is still important. Note that a decrease of the RGM2 irreducible error could be expected by using $\left\{\frac{\partial}{\partial x_{i}}\left(\bar{S}_{i j}^{\ominus} \frac{\partial \bar{Z}}{\partial x_{j}}\right) ; \frac{\partial}{\partial x_{i}}\left(\bar{\Omega}_{i j} \frac{\partial \bar{Z}}{\partial x_{j}}\right)\right\}$ as set of variables. But this would imply to further decompose RGM2 in two terms with two distinct coefficients to evaluate, leading to a more complex model. Due to the encouraging performance of RGM1, our proposition is just to keep the RGM1 formulation (8) to regularize the gradient model and to propose a dynamic procedure to compute the model coefficient. The dynamic procedure is expected improve the functional performance of the model, with a better evaluation of the mean SGS scalar dissipation, and the structural performance, with a quadratic error closer to the irreducible error.

\section{B. Dynamic procedure applied to the regularized gradient model RGM1}

The performances of RGM1 was measured by keeping a static coefficient coming from a Taylor series expansion. We now propose a dynamic procedure to improve the model performances. The regularized gradient model is thus re-written as,

$$
T_{i}^{\mathrm{RGM} 1}=C \bar{\Delta}^{2} \bar{S}_{i j}^{\ominus} \frac{\partial \bar{Z}}{\partial x_{j}},
$$

with $C$ a coefficient to evaluate. The dynamic procedure uses a test filter, noted $\hat{\imath}$, defined similarly to the first one, with a filter size such as $\hat{\Delta}=2 \bar{\Delta}$. Before describing this dynamic procedure, the mathematical derivation of the gradient model can be first recalled. The starting point is to write a Taylor series expansion for the filtering operation to evaluate $\overline{u_{i} Z}$ as function of $\bar{u}_{i}$ and $\bar{Z}$ (see Bedford and Yeo ${ }^{17}$ for details). This leads to (keeping only the first term of the Taylor series),

$$
\overline{u_{i} Z}=\bar{u}_{i} \bar{Z}+\frac{\bar{\Delta}^{2}}{12} \frac{\partial \bar{u}_{i}}{\partial x_{j}} \frac{\partial \bar{Z}}{\partial x_{j}}+\mathcal{O}\left(\bar{\Delta}^{4}\right) .
$$

The gradient model is obtained by neglecting the terms with an order higher than $\bar{\Delta}^{2}$ in the RHS. 
The proposed dynamic procedure is based on similar Taylor series expansions applied at the test filter level as already done in previous works ${ }^{22,32}$. Indeed, the consideration used to derive the gradient model, Eq. (13), can now be done also at the test filter level. The Taylor series expansion of the test filter for $f$ and $g$, both quantities describing flow fields, leads to

$$
\widehat{f g}-\hat{f} \hat{g}=\frac{\hat{\Delta}^{2}}{12} \frac{\partial \hat{f}}{\partial x_{j}} \frac{\partial \hat{g}}{\partial x_{j}}+\mathcal{O}\left(\hat{\Delta}^{4}\right) .
$$

By taking, $f=\bar{u}_{i}$ and $g=\bar{Z}$, Eq. (14) writes,

$$
\widehat{\bar{u}_{i} \overline{\bar{Z}}}-\hat{\bar{u}}_{i} \hat{\bar{Z}}=\frac{\hat{\Delta}^{2}}{12} \frac{\partial \hat{\bar{u}}_{i}}{\partial x_{j}} \frac{\partial \hat{\bar{Z}}}{\partial x_{j}}+\mathcal{O}\left(\hat{\Delta}^{4}\right) .
$$

Now, neglecting the terms with an order higher than $\hat{\Delta}^{2}$ and using the proposed regularization with the same dynamic coefficient, the model at the test filter level writes,

$$
\widehat{\bar{u}_{i} \bar{Z}}-\hat{\bar{u}}_{i} \hat{\bar{Z}}=C \hat{\Delta}^{2} \hat{\bar{S}}_{i j}^{\ominus} \frac{\partial \hat{\bar{Z}}}{\partial x_{j}} .
$$

This defines a relation between the Leonard-type term, $L_{i}=\widehat{\bar{u}_{i} \bar{Z}}-\hat{\bar{u}}_{i} \hat{\bar{Z}}$, and other quantities available in LES. This relation can thus be used to compute the model coefficient $C$. Assuming $C$ constant over homogeneous directions, it can be evaluated from a least-squares approximation according to Lilly's method ${ }^{11}$. The new dynamic procedure is now defined as

$$
C_{c}=\frac{\left\langle L_{i} N_{i}\right\rangle}{\left\langle N_{i} N_{i}\right\rangle}
$$

where $N_{i}=\hat{\Delta}^{2} \hat{\bar{S}}_{i j}^{\ominus} \partial \hat{\bar{Z}} / \partial x_{i}$ and where the brackets indicate a statistical average over homogeneous directions of the flow. Eq. (12) and (17) define the dynamic regularized gradient model, referred as DRGM in what follows. Note that similar dynamic procedures, based on Taylor series expansions, have been already used with success to model the SGS scalar variance $^{32}$ or to improve the accuracy of mixed model ${ }^{22}$.

The DRGM performances are now measured and compared with performance of the dynamic eddy diffusivity mode (DEDM), of the gradient model (GM) and of the static regularized model, Eq. (8), now noted RGM. Again, the optimal estimation analysis is performed to evaluate the capacity of the new model to accurately predict the divergence of the SGS scalar flux, $\partial T_{i} / \partial x_{i}$. Figure 6 shows the comparison of the model errors. Note that DRGM and RGM have the same irreducible errors because these models are based on 


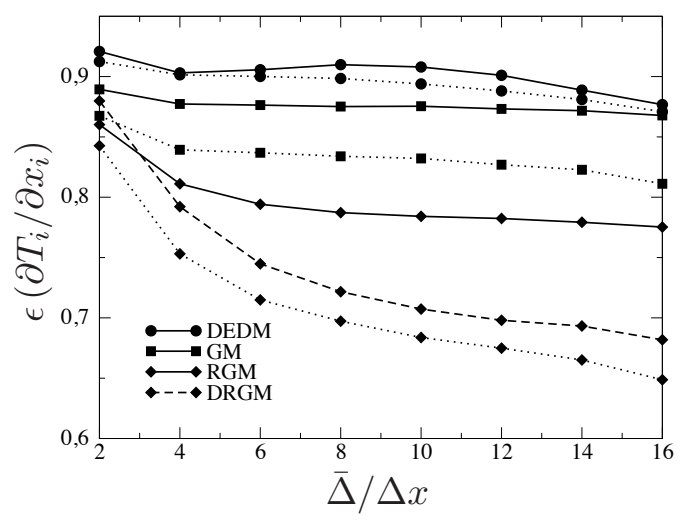

FIG. 6. Quadratic errors (QE) and irreducible errors (IE) as a function of the filter width for the proposed dynamic procedure (DRGM). The results for DEDM, GM and the standard (static) regularized gradient model (RGM) are shown for comparison. The dotted lines show the corresponding irreducible errors. Note that DRGM and RGM have the same irreducible error.

the same set of variables. The DRGM quadratic errors are the smallest quadratic errors, showing the improvement allowed by the dynamic procedure. This shows a significant improvement of the structural performance of the regularized model in comparison with the static formulation. Moreover, the DRGM quadratic error stays close to its irreducible errors, showing that it would be difficult to improve the model without adding new variables.

The functional performance can also been studied. Figure 7 shows the evolution of the mean SGS scalar dissipation with the filter width. The improvement of the functional performance of the dynamic procedure is characterized by an increasing of the SGS scalar dissipation magnitude for DRGM. This allows to correct the under-prediction of the GS/SGS transfer observed with RGM.

\section{A POSTERIORI (LES) TESTS}

\section{A. Description of the LES test cases}

The dynamic regularized gradient model (DRGM) for $T_{i}$ has been implemented and is now tested by performing large-eddy simulations (LES). The model is compared with the dynamic eddy diffusivity model (DEDM) and the gradient model (GM). Two flow configurations are 


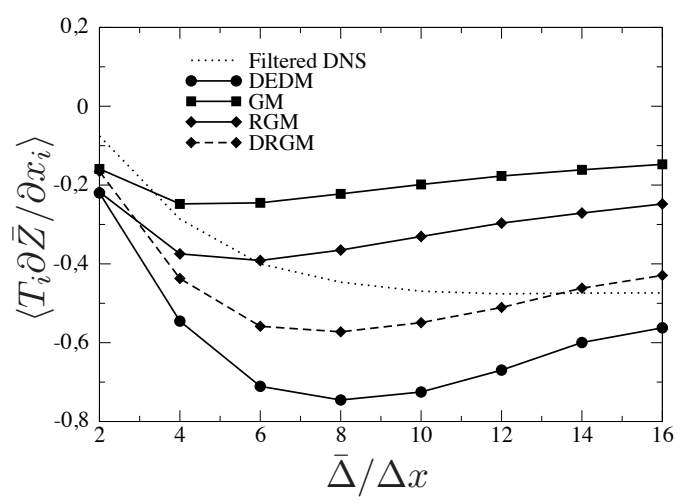

FIG. 7. Mean SGS dissipation as a function of the filter width for tfor for the proposed dynamic procedure (DRGM). The results for DEDM, GM and the standard (static) regularized gradient model (RGM) are shown for comparison. The dotted line show the SGS dissipation given by the filtered DNS data.

tested. The first case consists to the forced homogeneous isotropic turbulence. The second case consists to a temporal turbulent plane jet. These configurations are solved by using spectral method. Note that in all these test cases, the velocity field is still solved by direct numerical simulation (DNS). Thus, at each time step, the velocity field is extrapolated from the DNS to the LES mesh in spectral space. This spectral extrapolation is equivalent to a spectral cut-off filter. The resulting filtered velocity field is then used to advance the filtered scalar field. The advantage of this procedure is that no SGS model is needed for the Navier-Stokes equations and there is then no modeling error on the velocity field used in the filtered scalar equation. Thus, when the LES data are compared with the filtered DNS data, the difference will only be due to the model used for the SGS scalar flux.

First, forced homogeneous isotropic turbulence (FHIT) test cases are considered. The first FHIT configuration is similar to the one used in the a priori tests : $R_{\lambda} \approx 180$ and $S c=0.7$. In this case, the velocity field is still resolved on $512^{3}$ grid points. Two LES meshes for the scalar fields are used to investigate the performance of the models: $64^{3}$ and $32^{3}$ grid points. Moreover, to evaluate the influence of the Reynolds number on the models performance, another LES case is considered with the same Schmidt number but with a higher Reynolds number, $R_{\lambda} \approx 300$, on a mesh composed by $64^{3}$ grid points. This case is compared with a DNS performed on $1024^{3}$ grid points. Finally, for $R_{\lambda} \approx 180$, a last LES case 
is studied for a higher Schmidt number, $S c=4$, on a mesh composed by $64^{3}$ grid points. For Schmidt number higher than one, the smallest mixing scale is given by the Batchelor scale, $\eta_{B}=\eta_{K} / S c^{1 / 2}$, with $\eta_{K}$ the Kolmogorov scale. For DNS of this last flow configuration, a higher resolution is thus needed for the scalar field than for the velocity fields. In this case, the LES results are thus compared with a DNS using $1536^{3}$ grid points for the scalar field and still $512^{3}$ grid points for the velocity field.

For the second test case, a temporal turbulent plane jet flow configuration is considered. The configuration is similar to the one studied by Silva and Pereira ${ }^{37}$. In this configuration, the computational domain is periodic in the three spatial directions. Thus, one studies the temporal evolution of the flow generated by an initial plane jet velocity profile. The initial velocity and scalar profiles are described by a classic hyperbolic-tangent profile ${ }^{37}$. For this configuration, the molecular viscosity, $\nu$, is defined to have the Reynolds number equal to $R e_{H}=\left(U_{1}-U_{2}\right) H / \nu=10,000$, where $H$ is the plane jet inlet slot width, $U_{1}$ is the initial jet velocity and $U_{2}$ is the co-flow velocity. The scalar value is initially 1 in the jet and 0 in the co-flow and the molecular Schmidt number is 0.7 . The computational box size is $\left(L_{x}, L_{y}, L_{z}\right)=(4 H, 6 H, 4 H)$, with $x, y$ and $z$, the streamwise, the normal and the spanwise directions, respectively. For the DNS, the grid size consists then in $N_{x}^{\mathrm{DNS}} \times N_{y}^{\mathrm{DNS}} \times N_{z}^{\mathrm{DNS}}=$ $1024 \times 1536 \times 1024$ grid points. The LES of the passive scalar is then performed on a mesh composed by $N_{x}^{\mathrm{LES}} \times N_{y}^{\mathrm{LES}} \times N_{z}^{\mathrm{LES}}=128 \times 192 \times 128$ grid points. The LES results will be compared to filtered DNS results to evaluate the models ability to deal with transition stages and mean shear regions.

\section{B. LES of isotropic homogeneous turbulence}

Figure 8 shows the time evolution of the LES resolved scalar variance, $\left\langle\bar{Z}^{\prime 2}\right\rangle=\langle\bar{Z} \bar{Z}\rangle-$ $\langle\bar{Z}\rangle\langle\bar{Z}\rangle$, in isotropic homogeneous turbulent flow. The LES resolved scalar variance is compared with the variance of the filtered scalar field coming from the DNS data. The DNS scalar variance (without filtering), $\left\langle Z^{\prime 2}\right\rangle=\left\langle Z^{2}\right\rangle-\langle Z\rangle^{2}$, are also shown for comparison. The fluctuations smaller than the filter width are not taken into account in the filtered scalar variance evaluation. This implies that the filtered scalar variance has to be smaller than the DNS scalar variance and that this gap is higher for higher filter width, Fig. 8 (a) and (b). For the same reason, the difference between the filtered scalar variance and the DNS scalar 


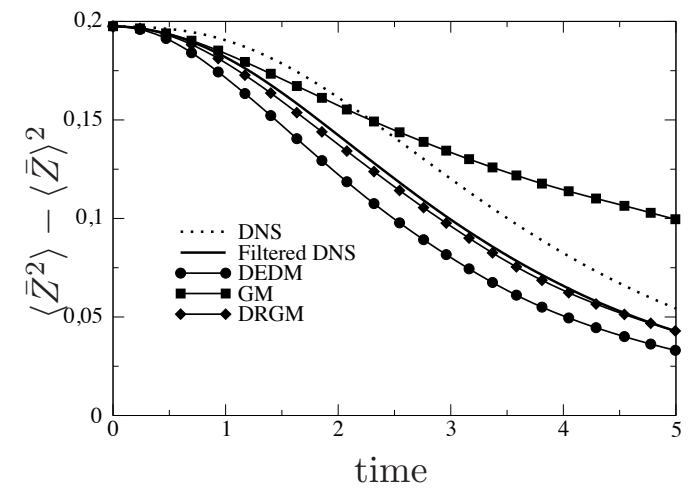

(a) $S c=0.7 ; R_{\lambda} \approx 180 ; 32^{3}$ grid points

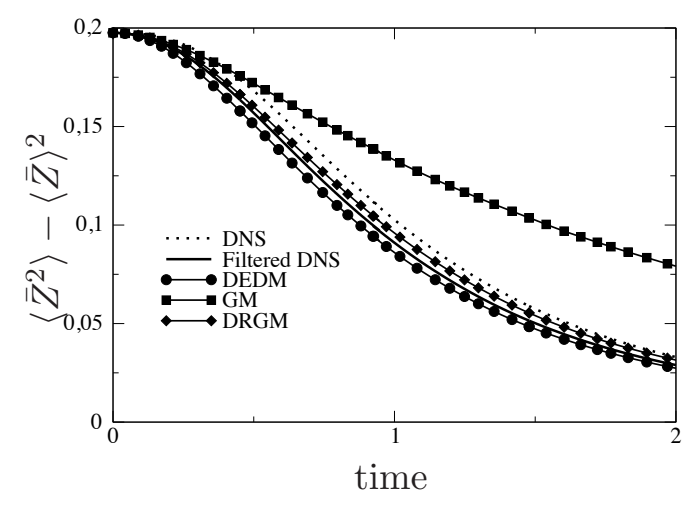

(c) $S c=0.7 ; R_{\lambda} \approx 300 ; 64^{3}$ grid points

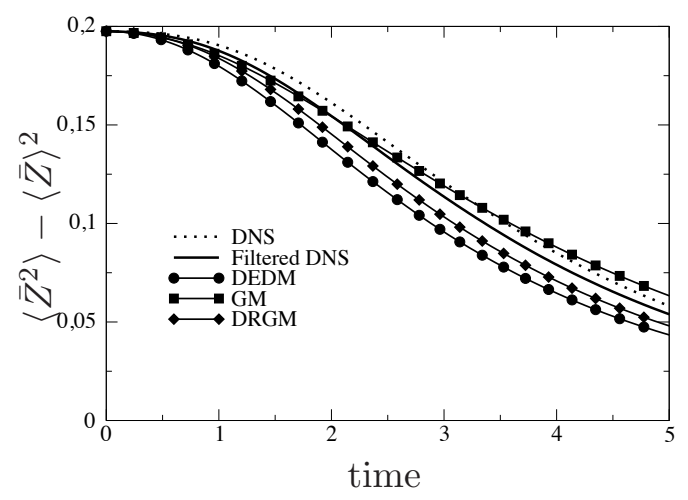

(b) $S c=0.7 ; R_{\lambda} \approx 180 ; 64^{3}$ grid points

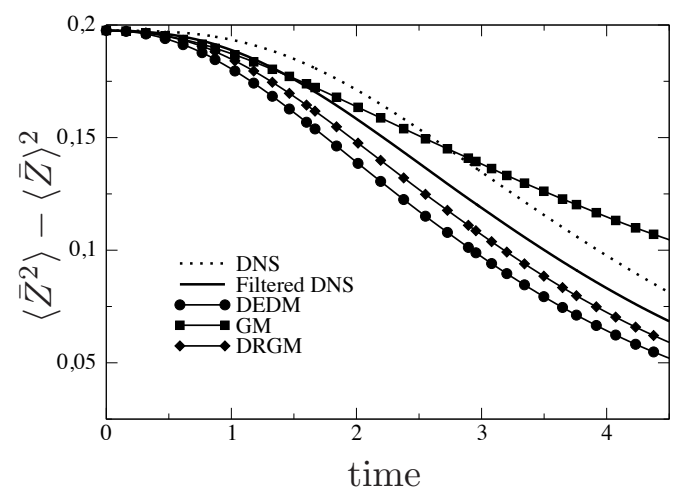

(d) $S c=4 ; R_{\lambda} \approx 180 ; 64^{3}$ grid points

FIG. 8. Evolution of the LES resolved scalar variance, $\left\langle\bar{Z}^{\prime 2}\right\rangle$, with time. The filtered and no-filtered DNS scalar variances are also shown for comparison.

variance is higher for the case with $S c=4$ because the Batchelor scale is smaller for this case and the part due to subfilter scales is more important, Fig. 8 (b) and (d). In all the cases, the same observations can be done about the models performance. First, the DEDM resolved scalar variance is always notably smaller than the filtered DNS scalar variance. This is due to an over-prediction of the SGS dissipation with this model as already found in the $a$ priori tests. Conversely, the GM resolved scalar variance can be even higher than the DNS (no-filtered) scalar variance. This characterizes the generation of non-physical fluctuations at the smallest resolved scales with this model. This is due to a large under-prediction of the SGS dissipation. As expected, DRGM allows to correct this behavior with a resolved scalar variance always smaller than the DNS scalar variance and close to the filtered scalar 


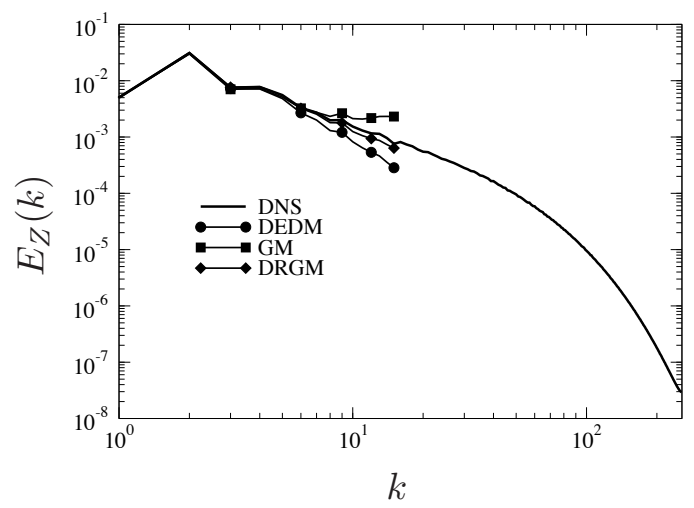

(a) $S c=0.7 ; R_{\lambda} \approx 180 ; 32^{3}$ grid points

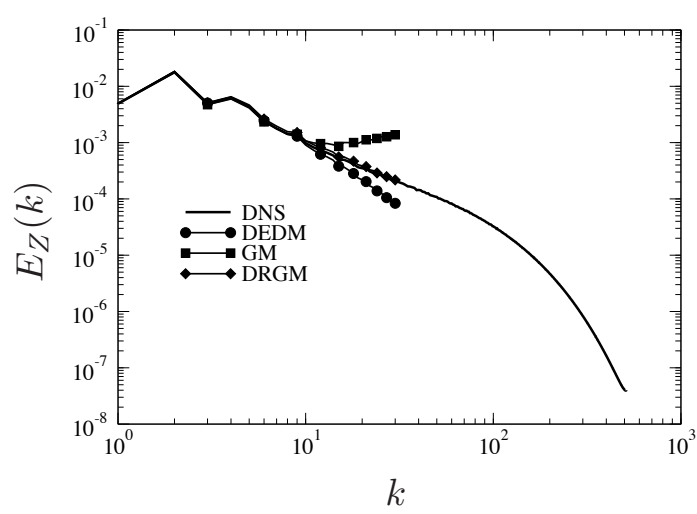

(c) $S c=0.7 ; R_{\lambda} \approx 300 ; 64^{3}$ grid points

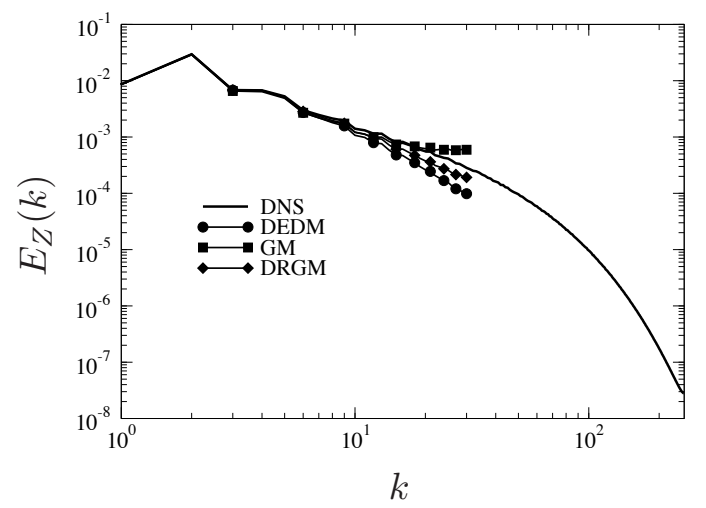

(b) $S c=0.7 ; R_{\lambda} \approx 180 ; 64^{3}$ grid points

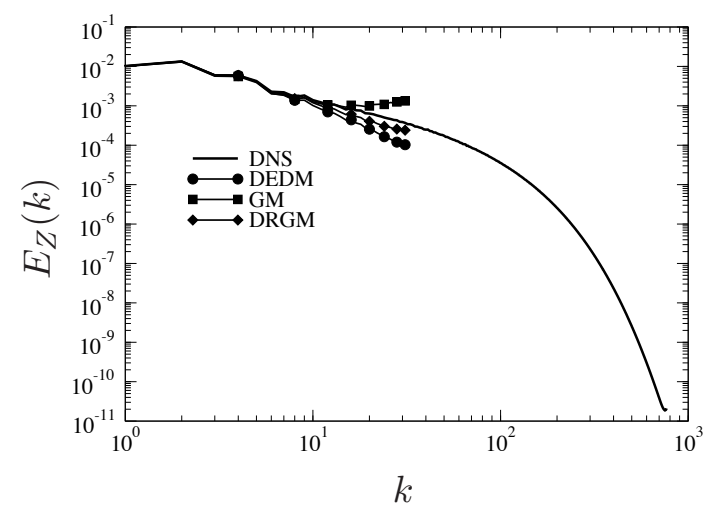

(d) $S c=4 ; R_{\lambda} \approx 180 ; 64^{3}$ grid points

FIG. 9. Scalar variance spectrum, $E_{Z}(k)$, when $\left\langle\bar{Z}^{\prime 2}\right\rangle \approx 0.1$.

variance. Moreover, DRGM has better behavior than DEDM with a weaker over-prediction of the decreasing of the resolved scalar variance than this classic model.

For further analysis, the scalar variance spectrum is shown in Fig. 9. The spectra are computed when the mixing is well established. The models performances are still similar in all the cases. First, as expected, GM leads to an over-prediction of the scalar variance spectrum at the smallest resolved scales (highest resolved wave numbers). This characterizes the unstable behavior of this model with the generation of non-physical fluctuations due to a over-estimation of the back-scatter effect. Conversely, DEDM under-predicts the scalar variance spectrum at the smallest resolved scales. This is due to the over-prediction of the SGS dissipation at these scales. DRGM allows to correct these behaviors and stays close to the DNS results, even for very coarse LES, Fig. 9 (a). Note that the models performance 


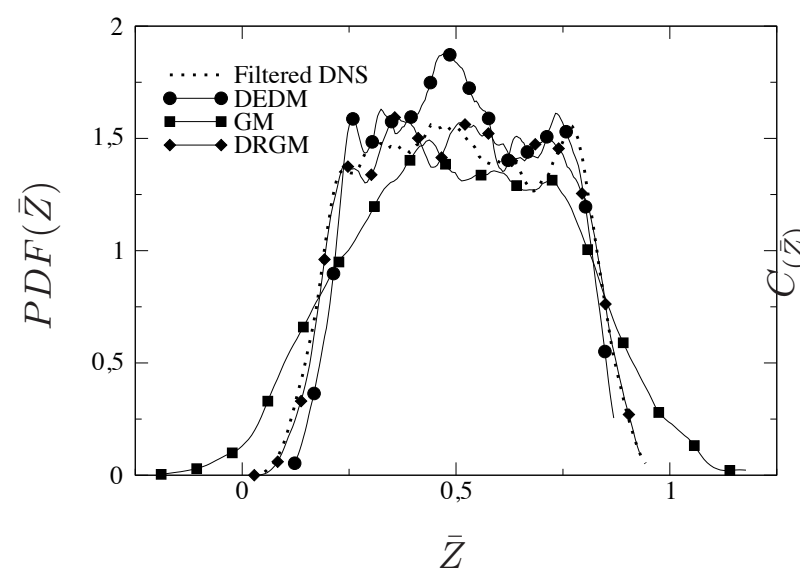

(a) Probability density function (PDF)

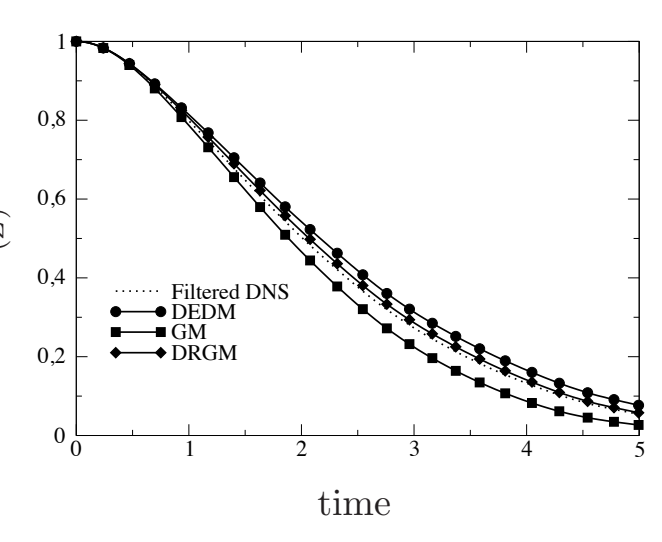

(b) Time correlation

FIG. 10. Additional statistics for LES on $32^{3}$ grid points at $R_{\lambda} \approx 180$ and $S c=0.7$ : Probability density function $(\mathrm{PDF})$ of the LES resolved scalar when $\left\langle\bar{Z}^{\prime 2}\right\rangle \approx 0.1$ (a) and time correlation of the LES resolved scalar, $C_{(\bar{Z})}$, computed by Eq. (18) (b). DNS filtered scalar results are also shown in dotted line for comparison.

is not influenced by the local turbulence of the flow and by the molecular scale transport properties in the range of the Reynolds and Schmidt numbers used. The same trend is found in all the LES performed.

Finally, the influence of the SGS scalar flow model can be studied from additional statistics. First, the scalar probability density function (PDF) is shown in Fig. 10 (a) when the mixing is established. The consequences of the SGS scalar flux model performance can then be observed on the local mixing prediction. First, the over-dissipation predicted by DEDM implies that the part of unmixed fluid, $\bar{Z} \approx 0$ or $\bar{Z} \approx 1$, is under-predicted with smaller PDF values than the filtered DNS, whereas the part of fully mixed fluid, $\bar{Z} \approx 0.5$, is over-predicted with higher PDF values than the filtered DNS. This characterizes a over-estimation of the mixing process as already observed with the decay of the scalar variance, Fig. 8. Moreover, the unstable behavior of GM is characterized by unphysical values of $\bar{Z}$. Indeed, for this model, there are non zero probabilities to find $\bar{Z}<0$ or $\bar{Z}>1$, whereas the scalar has to be bounded between its initial values, 0 and 1 . This behavior will lead to modeling problem when the scalar represents chemical species concentration or temperature in practical applications. The new proposed model allows to correct this problem. Indeed, it can be seen that the PDF computed from the LES using DRGM is close to the PDF computed from 

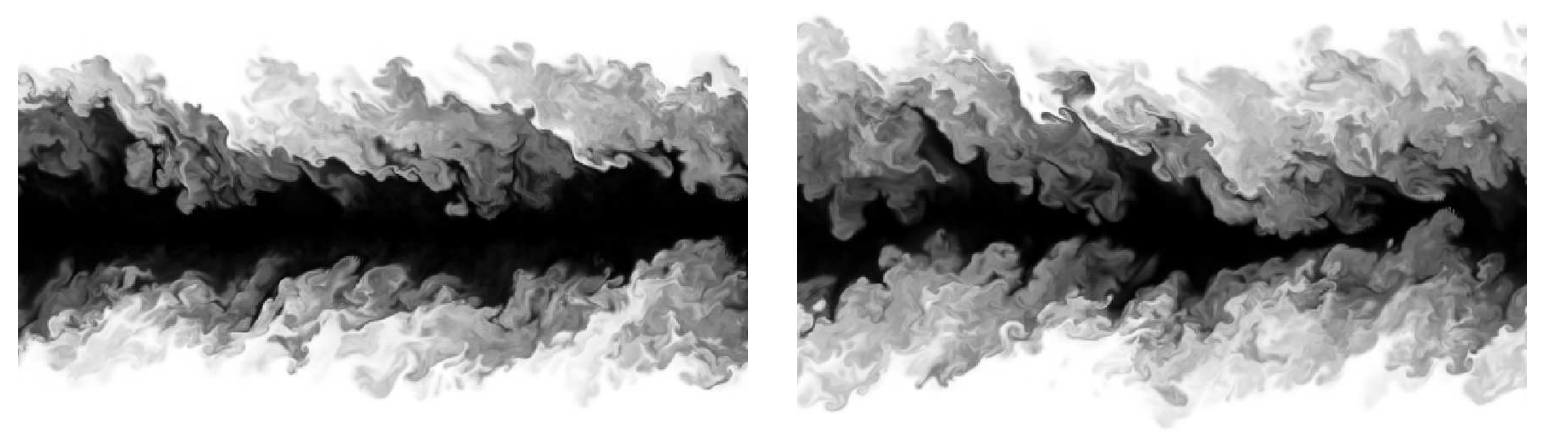

FIG. 11. Contour of scalar from DNS results for two time during the plane jet transition toward a turbulent state. The scalar is between 0 (white) and 1 (black)

the filtered DNS data. Finally, we study the time correlation of the LES resolved scalar. Indeed, subgrid-scales contribute to temporal decorrelation of the resolved scales. Thus, a SGS model has to model the subgrid-scales effects, and has also to reproduce as well as possible this temporal decorrelation ${ }^{38}$. The time auto-correlation of the LES resolved scalar is defined as

$$
C_{(\bar{Z})}(t)=\frac{\left\langle\bar{Z}^{\prime}\left(\vec{x}, t_{0}\right) \bar{Z}^{\prime}(\vec{x}, t)\right\rangle}{\left(\left\langle\bar{Z}^{\prime 2}\left(\vec{x}, t_{0}\right)\right\rangle\left\langle\bar{Z}^{\prime 2}(\vec{x}, t)\right\rangle\right)^{1 / 2}},
$$

where $\bar{Z}^{\prime}$ is the scalar fluctuation field. Figure 10 (b) shows the evolution of $C_{(\bar{Z})}$ for the different LES. The time correlation for filtered DNS data are also shown for comparison. DRGM seems to lead to better agreement with the filtered DNS data. Indeed, DEDM lead to higher correlations whereas GM predicts faster decorrelation than the other models. This is probably due to the prediction of the mixing activities at the smallest resolved scales, as already shown with scalar variance spectra. Thus, the over-prediction of the fluctuations at these smallest resolved scales by GM leads to an over-prediction of the temporal decorrelation. Conversely, the under-prediction of the mixing activities at these scales leads to a weak under-prediction of the decorrelation process.

\section{LES of turbulent plane jet}

From the previous section, the new proposed dynamic regularized gradient model (DRGM) shows good performances in comparison with other classic SGS models. In par- 

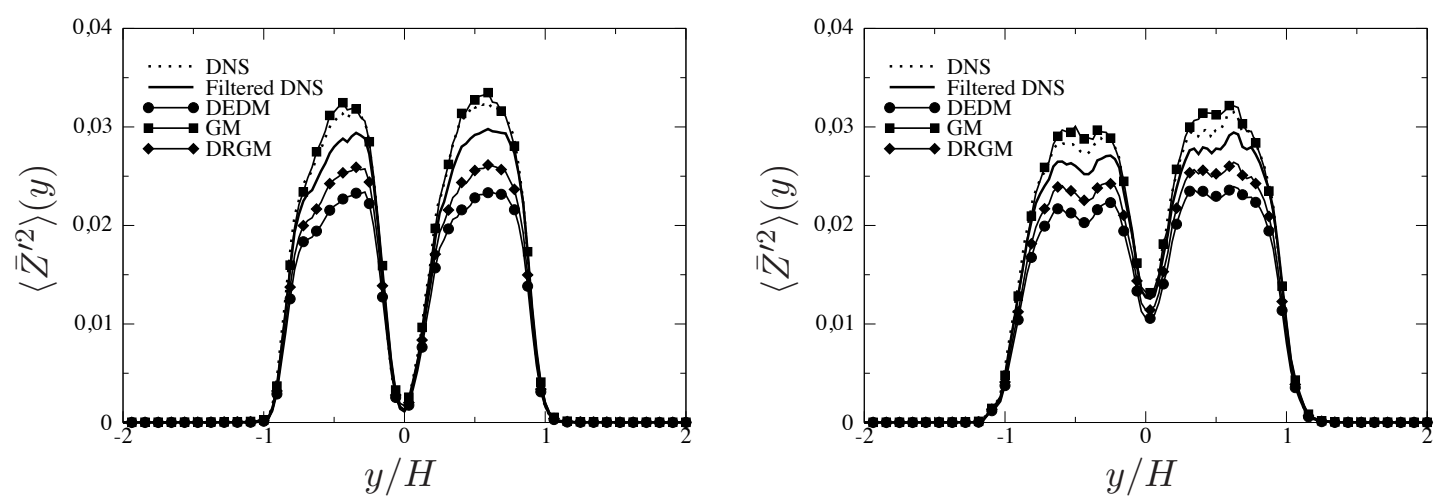

FIG. 12. Profile of the LES resolved scalar variance, $\left\langle\bar{Z}^{\prime 2}\right\rangle$ for two times during the plane jet transition toward a turbulent state (corresponding to Fig. 11). The filtered and no-filtered DNS scalar variances are also shown for comparison.

ticular, the regularization allows to correct the unphysical behavior found with the classic gradient model. Now, to test the ability of the new model to deal with transition stages and shear regions, LES of a temporal plane jet are performed using DEDM, GM and DRGM. For DEDM and DRGM, the dynamic procedure used a plan averaging over both homogeneous directions, $x$ and $z$. The results are compared with filtered DNS data for two time during the plane jet transition toward a turbulent state. Figure 11 shows the contour of scalar for these two times to illustrates the mixing stages. The turbulent mixing develops thanks to coherent vortices. First, the Kelvin-Helmholtz vortices dominates the transition process. Then, streamwise vortices appear leading to a tridimensionalization of the jet and to an abrupt transition towards a developed turbulent state in agreement with the classical scenario of transition in free shear layers ${ }^{39}$.

First, figure 12 shows the LES resolved scalar variance profile, $\left\langle\bar{Z}^{\prime 2}\right\rangle(y)$. The results are compared with the variance of the filtered scalar field coming from the DNS data, and the DNS scalar variance (without filtering) profile are also shown. The resolved scalar variance is first higher in the shear layers, $|y / H| \approx 0.5$, where the first turbulent scales develop. Then, the resolved scalar variance grows in the middle of the jet, $y / H=0$, due to the merging of the shear layers and the development of turbulent scales in the jet core. In this flow configuration, the models performance results are similar to the results obtained in FHIT cases. Indeed, the GM resolved scalar variance is higher than the DNS (no-filtered) scalar 

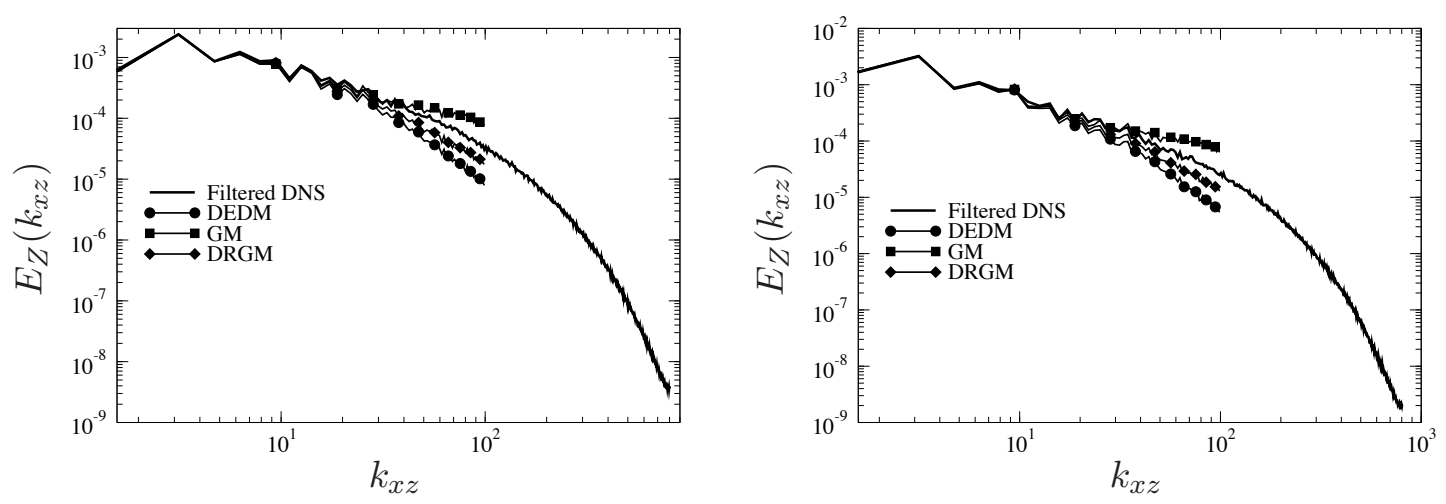

FIG. 13. 2D scalar variance spectrum, $E_{Z}\left(k_{x z}\right)$, in the middle of the shear layer $(y / H=0.5)$ for two time during the plane jet transition toward a turbulent state (corresponding to Fig. 11).

variance, characterizing the generation of non-physical mixing scales. Moreover, the overprediction of the SGS dissipation with DEDM leads to a smaller value of the resolved scalar variance with this model. DRGM allows a better prediction of the resolved scalar variance, avoiding the GM unphysical behavior. However, even if DRGM is in better agreement with filtered DNS data than DEDM, it can be observed that DRGM has a significant underprediction to the variance. This is due to an over-prediction of the SGS dissipation at the smallest resolved scales as shown by Fig. 13. Additional works will test the influence of the averaging process used for the dynamic procedure ${ }^{40}$ on this behavior. Finally, figure 13 leads to similar conclusions to the FHIT cases: GM leads to an over-prediction of the scalar variance spectrum and DEDM significantly under-predicts the scalar variance spectrum at the smallest resolved scales.

\section{CONCLUSION}

In this paper, we have introduced a closure for predicting subgrid-scale (SGS) flux of a passive scalar in the context of large eddy simulation (LES). The proposed closure is derived from the gradient model (GM) which is based on a Taylor series expansion of the filtering operation. The GM is known to provide good estimations of local SGS fluxes in a priori tests (i.e. good structural performance). Still the GM yields unstable behavior in a posteriori tests because of its predicting incorrect grid scale/subgrid scale (GS/SGS) 
transfers of scalar variance (weak functional performance). This weak performance is due to an over-prediction of resolved scalar variance through too large backscattering from the SGS. In order to propose a physically based regularization of the GM, a decomposition of the velocity gradient has been performed to split the gradient model into three terms respectively associated with compression, stretching and rotation. Thus, while the GM is shown to systematically decrease the resolved scalar variance in local compression cases, it is responsible for increasing the resolved scalar variance in local stretching cases. The GM prediction of the resolved velocity gradient effects on the GS/SGS transfers of scalar variance is consistent with behaviors observed on a simple flow. A series of a priori tests and optimal estimator predictions shows that a regularized model with good performance can be build from the GM by retaining only the term associated with compression. A dynamic procedure is then applied to the regularized version of the gradient model. Various a posteriori tests with the proposed dynamic regularized gradient model (DRGM) indicate that the model provides good estimation of GS/SGS transfers. As compared to classical eddy diffusivity models, the DRGM also improves substantially scalar variance spectra and the probability density function of scalar distributions. Note that this work has been performed by using spectral method to be able to neglect numerical and modeling errors interaction and to focus only on the modeling errors. Future works will be devoted to consider the model in the case where numerical errors can not be neglected. This is an important step to evaluate the ability of the model to be used in industrial and geophysical applications.

\section{ACKNOWLEDGMENTS}

This work is supported by the Agence Nationale pour la Recherche (ANR) under Contract No. ANR-2010-JCJC-091601. This work was performed using HPC resources from GENCIIDRIS (Grant 2012-020611). The authors thankfully acknowledge the hospitality of the Center for Turbulence Research, NASA-Ames and Stanford University, where a part of this work has been done during the Summer Program 2012. 


\section{APPENDIX: ANALYTICAL DECOMPOSITION OF THE FILTERED STRAIN RATE TENSOR}

In order to guide practical applications of the Dynamic Regularized Gradient Model, we provide in this appendix information about the decomposition of the filtered strain rate tensor. For the tests presented in this paper, the decomposition of $\bar{S}_{i j}$ according to equation (5) was performed numerically with the help of the LAPACK library (http://www.netlib.org/lapack/).

\section{Eigenvalues of the filtered strain rate tensor in $3 \mathrm{D}$ case}

The filtered strain rate tensor, $\bar{S}_{i j}$, being symmetric, it has real eigenvalues. These eigenvalues can be obtained analytically by solving the characteristic polynomial with the Cardano's method. The $k^{\text {th }}$ eigenvalue, $\lambda^{(k)}$, is thus given by

$$
\lambda^{(k)}=2 \sqrt{-\frac{c}{3}} \cos \left(\frac{1}{3} \cos ^{-1}\left(-\frac{b}{2} \sqrt{-\frac{27}{c^{3}}}\right)+\frac{2 k \pi}{3}\right),
$$

where

$$
c=-\frac{1}{2} \bar{S}_{i j} \bar{S}_{i j}
$$

and

$$
b=\bar{S}_{13}^{2} \bar{S}_{22}+\bar{S}_{12}^{2} \bar{S}_{33}+\bar{S}_{23}^{2} \bar{S}_{11}-\bar{S}_{11} \bar{S}_{22} \bar{S}_{33}-2 \bar{S}_{12} \bar{S}_{13} \bar{S}_{23}
$$

The eigenvalues determination allows thus to write the linear system needed to obtain the eigenvectors.

\section{Full decomposition of the filtered strain rate tensor in $2 \mathrm{D}$ case}

For two dimensional applications of the regularization presented in this paper, an explicit analytical expression of RGM1 can be readily obtained. Indeed, it can be shown that the eigenvalues of $\bar{S}_{i j}$ in two dimensions are respectively :

$$
\lambda_{+}=\frac{1}{2}\left(d+\sqrt{a^{2}+r^{2}}\right) \quad \lambda_{-}=\frac{1}{2}\left(d-\sqrt{a^{2}+r^{2}}\right)
$$

where $r=\partial \bar{v} / \partial x+\partial \bar{u} / \partial y$ is the rate of filtered shear strain, $a=\partial \bar{u} / \partial x-\partial \bar{v} / \partial y$ is the rate of filtered normal strain and $d=\partial \bar{u} / \partial x+\partial \bar{v} / \partial y$ is the horizontal filtered divergence, with 
$\bar{u}$ and $\bar{v}$ the two dimensional filtered velocity component in $x$ and $y$ direction, respectively. Straighforward algebra then yields the eigenvectors of $\bar{S}_{i j}$. Finally, equation (5) allows to decompose $\bar{S}_{i j}=\bar{S}_{i j}^{\oplus}+\bar{S}_{i j}^{\ominus}$ with

$$
\bar{S}_{i j}^{\oplus}=\frac{1+\delta}{4}\left(\begin{array}{cc}
p_{+} & r \\
r & q_{+}
\end{array}\right) \quad \bar{S}_{i j}^{\ominus}=\frac{1-\delta}{4}\left(\begin{array}{cc}
p_{-} & r \\
r & q_{-}
\end{array}\right)
$$

where the following notations have been used

$$
\begin{array}{cc}
p_{+}=\sqrt{a^{2}+r^{2}}+a & q_{+}=\sqrt{a^{2}+r^{2}}-a \\
p_{-}=-\sqrt{a^{2}+r^{2}}+a & q_{-}=-\sqrt{a^{2}+r^{2}}-a
\end{array}
$$

and $\delta=d / \sqrt{a^{2}+r^{2}}$. The bidimensional model proposed by Le Sommer at al. ${ }^{28}$ can be related to the above decomposition.

1 H. Pitsch. Large-eddy simulation of turbulent combustion. Ann. Rev. Fluid Mech., 38:453-482, 2006.

2 B. Fox-Kemper and D. Menemenlis. Can large eddy simulation techniques improve mesoscale rich ocean models? In Matthew W. Hecht and Hiroyasu Hasumi, editors, Geophysical Monograph Series, volume 177, pages 319-337. American Geophysical Union, Washington, D. C., 2008.

3 M. Lesieur and O. Métais. New trends in large-eddy simulations of turbulence. Ann. Rev. Fluid Mech., 28:45-82, 1996.

4 C. Meneveau and J. Katz. Scale-invariance and turbulence models for large-eddy simulation. Ann. Rev. Fluid Mech., 28:45-82, 2001.

${ }_{5}$ P. Sagaut. Large eddy simulation for incompressible flows : an introduction. Springer, 2006.

6 M. Lesieur and R. Rogallo. Large eddy simulation of passive scalar diffusion in isotropic turbulence. Phys. Fluids A, 1(9):718-722, 1989.

7 O. Métais and M. Lesieur. Spectral large-eddy simulation of isotropic and stably stratified turbulence. J. Fluid Mech., 239:157-194, 1992.

8 C. Brun, G. Balarac, C. B. da Silva, and O. Métais. Effects of molecular diffusion on the subgrid-scale modelling of passive scalars. Phys. Fluids, 20(2), 2008. 
9 P. Moin, K. Squires, W. Cabot, and S. Lee. A dynamic subgrid-scale model for compressible turbulence and scalar transport. Phys. Fluids A, 3:2746-2757, 1991.

10 M. Germano, U. Piomelli, P. Moin, and W. H. Cabot. A dynamic subgrid-scale eddy viscosity model. Phys. Fluids A, 3, 1991.

11 D.K. Lilly. A proposed modification of the germano subgrid-scale closure method. Phys. Fluids A, 4:633-635, 1992.

12 R. A. Clark, J. H. Ferziger, and W. C. Reynolds. Evaluation of subgrid-scale models using an accurately simulated turbulent flow. J. Fluid Mech., 91:1-16, 1979.

13 C. W. Higgins, M. B. Parlange, and C. Meneveau. Turbulent heat flux and temperature gradient alignments in the lower atmosphere. Geophys. Res. Letts., 31(L22105), 2004.

14 S. Chumakov. A priori study of subgrid-scale flux of a passive scalar in isotropic homogeneous turbulence. Phys. Rev. E, 78(036313), 2008.

15 J. Bardina, J.H. Ferziger, and W.C. Reynolds. Improved subgrid scale models for large eddy simulation. AIAA Paper, 80-1357, 1980.

16 S. G. Chumakov and C. J. Rutland. Dynamic structure subgrid-scale models for large eddy simulation. Int. J. Numer. Meth. Fluids, 47:911-923, 2005.

17 K. W. Bedford and W. K. Yeo. Conjective filtering procedures in surface water flow and transport. Large Eddy Simulation of Complex Engineering and Geophysical Flows, edited by B. Galperin, and S. A. Orszag, Cambridge Univ. Press, New York, 1993.

18 T. Dubos and A. Babiano. Two dimensional cascades and mixing : a physical space approach. Journal of Fluid Mechanics, 467:81-100, 2002.

19 A. Leonard. On the energy cascade in large-eddy simulations of turbulent flows. Adv. in Geophys., A18:237, 1974.

20 H. Lu and Porté-Agel. A modulated gradient model for scalar transport in large-eddy simulation of the atmospheric boundary layer. Phys. Fluids, 25(015220), 2013.

21 Bert Vreman, Bernard Geurts, and Hans Kuerten. Large-eddy simulation of the turbulent mixing layer. Journal of Fluid Mechanics, 339:357-390, May 1997.

22 Y. Fabre and G. Balarac. Development of a new dynamic procedure for the clark model of the subgrid-scale scalar flux using the concept of optimal estimator. Phys. Fluids, 23(11), 2011.

23 C. Jiménez, F. Ducros, B. Cuenot, and B. Bédat. Subgrid scale variance and dissipation of a scalar field in large eddy simulations. Phys. Fluids, 13:1748-1754, 2001. 
24 C. B. da Silva and J. C. F. Pereira. Analysis of the gradient-diffusion hypothesis in large-eddy simulations based on transport equations. Phys. Fluids, 19:1-20, 2007.

25 H. S. Kang and C. Meneveau. Universality of large eddy simulation model parameters across a turbulent wake behind a heated cylinder. J. of Turbulence, 2002.

${ }^{26}$ K. K. Nomura and G. K. Post. The structure and dynamics of vorticity and rate of strain in incompressible homogeneous turbulence. J. Fluid Mech., 377:65-97, 1998.

27 B. Vreman, B. Geurts, and H. Kuerten. Large eddy simulation of the temporal mixing layer using the clark model. Theor. Comput. Fluid Dyn., 8, 1996.

28 J. Le Sommer, F. dOvidio, and G. Madec. Parameterization of subgrid stirring in eddy resolving ocean models. part 1: Theory and diagnostics. Ocean Modelling, 39:154169, 2011.

29 G.-H. Cottet and A. A. Wray. Anisotropic grid-based formulas for subgrid-scale models. Annual Research Brief, CTR, Stanford Univ., 1997.

30 K. Alvelius. Random forcing of three-dimensional homogeneous turbulence. Phys. Fluids, 11:1880-1889, 1999.

31 V. Eswaran and S.B. Pope. Direct numerical simulations of the turbulent mixing of a passive scalar. Phys. Fluids, 31:506-520, 1988.

32 G. Balarac, H. Pitsch, and V. Raman. Development of a dynamic model for the subfilter scalar variance using the concept of optimal estimators. Phys. Fluids, 20(3), 2008.

33 G. Balarac, H. Pitsch, and V. Raman. Modeling of the subfilter scalar dissipation rate using the concept of optimal estimators. Phys. Fluids, 20(9), 2008.

34 R. Deutsch. Estimation Theory. Prentice-ttall, Englewood Cliffs, N. J., 1965.

35 A. Moreau, O. Teytaud, and J. P. Bertoglio. Optimal estimation for large-eddy simulation of turbulence and application to the analysis of subgrid models. Phys. Fluids, 18:1-10, 2006.

36 J. A. Langford and R. D. Moser. Optimal LES formulations for isotropic turbulence. J. Fluid Mech., 398:321-346, 1999.

37 C.B. da Silva and J.C.F. Pereira. Invariants of the velocity-gradient, rate-of-strain, and rateof-rotation tensors across the turbulent/nonturbulent interface in jets. Phys. Fluids, 20(05), 2008.

38 G.W. He, R. Rubinstein, and L.-P. Wang. Effects of subgrid-scale modeling on time correlations in large eddy simulation. Phys. Fluids, 14:3186-2193, 2002. 
39 L. P. Bernal and A. Roshko. Streamwise vortex structure in plane mixing layers. J. Fluid Mech., $170,1986$.

40 C. Meneveau, T. Lund, and W. Cabot. A Lagrangian dynamic subgrid-scale model of turbulence. J. Fluid Mech., 319:353-385, 1996. 